\title{
Power Allocation for Cooperative Systems with Training-Aided Channel Estimation
}

\author{
Berna Gedik, Osama Amin, Student Member, IEEE, and Murat Uysal, Senior Member, IEEE
}

\begin{abstract}
Cooperative communication techniques promise the advantages of multi-input multi-output (MIMO) communications for wireless scenarios with single-antenna terminals. A main assumption in majority of the research work on cooperative communications is the availability of channel state information at the receiver. In practice, knowledge of the channel is obtained by sending known training (pilot) symbols to the receiver. In this paper, we study the effect of training on the performance of an amplify-and-forward cooperative relaying system with pilot-assisted channel estimator over quasi-static Rayleigh fading channels. We consider average received signal-to-noise ratio at the destination node as the objective function and formulate optimization problems for a single-relay scenario under total network power (TNP) and individual node power (INP) constraints. We aim to answer the following fundamental questions: Q1) How should overall transmit power be shared between training and data transmission periods?; Q2) How should training power be allocated to broadcasting and relaying phases?; Q3) How should data power be allocated to broadcasting and relaying phases? Our simulation results demonstrate that optimized schemes significantly outperform the original schemes with equal power allocation. Depending on the relay location, performance gains up to $5.5 \mathrm{~dB}$ and $2.8 \mathrm{~dB}$ are observed, respectively, under TNP and INP constraints.
\end{abstract}

Index Terms-Cooperative transmission, channel estimation, power allocation.

\section{INTRODUCTION}

C OOPERATIVE communication techniques [1]-[5] have emerged as a powerful alternative to reap the benefits of MIMO (multiple-input multiple output) communications in a wireless scenario with single-antenna terminals. Cooperative communication takes advantage of the broadcast nature of wireless transmission and creates a virtual antenna array through relaying among the cooperating nodes. Two main relaying techniques are amplify-and-forward $(\mathrm{AaF})$ and decodeand-forward $(\mathrm{DaF})$. In DaF relaying, the relay node decodes the signal received from the source, re-encodes (possibly with a different codebook), and forwards it to the destination. In AaF relaying, the relay normalizes the received signal with a proper scaling factor (without any attempt to decode it) and retransmits it to the destination. AaF relaying is preferable in analog systems and, since it avoids decoding, it is particularly

Manuscript received October 16, 2008; revised March 31, 2009; accepted May 26, 2009. The associate editor coordinating the review of this paper and approving it for publication was J. Luo.

This paper was presented in part at IEEE VTC'08 Fall, Calgary, Alberta, Canada, September 2008. The work of O. Amin is supported by the Egyptian Higher Education Ministry.

B. Gedik is with General Electric.

O. Amin and M. Uysal are with the Department of Electrical and Computer Engineering, University of Waterloo, Waterloo, ON, N2L3G1, Canada (email: \{oamin,muysal\}@ece.uwaterloo.ca).

Digital Object Identifier 10.1109/TWC.2009.081382 useful when the application is time-sensitive such as in the case of voice or live-video transmission [6].

Although cooperative communication has been relatively a new research area, there already exists a rich literature on the topic. However, a main underlying assumption in majority of the current literature is the availability of the channel state information (CSI) at the receiver. For a coherent receiver, the fading channel coefficients need to be estimated for detection stage. In DaF relaying, both relay and destination nodes need to be equipped with channel estimators which they use for the estimation of source-to-relay and relay-to-destination channels, respectively. In AaF relaying, the relay node might be designed with or without a channel estimator based on the adopted scaling factor [7], [8]. For the case of blind relays, the cascaded channel connecting the source and the destination via the relay should be estimated at the destination node. For the case of CSI-assisted relays, the estimation of the cascaded channel can be disintegrated into separate estimations of source-to-relay and relay-to-destination channels through the injection of a clean pilot symbol at the relay. However, such an approach would require additional pilot symbols, therefore reduce the bandwidth and power efficiency. It would be also necessary to quantize and transmit sourceto-relay channel estimate from the relay to the destination. Quantization errors and transmission reliability of the feedforward control channel would further degrade the quality of channel estimate in practical implementation. We therefore consider blind relays in our paper.

Related Literature and Contributions of Our Work: Coherent maximum likelihood (ML) receiver with imperfect channel estimation (i.e., mismatched-coherent receiver) for AaF relaying has been studied in [9] and [10]. In [9], Mheidat and Uysal investigate the performance of mismatchedcoherent receiver for a distributed space-time block code over both quasi-static and time-varying fading channels. In [10], Patel and Stuber consider a multi-hop relay scenario. They derive a channel estimator for cascaded Rayleigh fading channel and analyze the bit error rate performance. No effort towards resource optimization for training/data transmission is made either in [9] or in [10]. In [11], Cho et al. address resource allocation problem (i.e., energy and relay location optimization) for a multi-relay network. However, they assume differential demodulation which avoids the need for channel estimation.

In [12], Wang et al. aim to optimize the training and data powers with average received signal-to-noise ratio (SNR) as their objective function. Their transmission scenario is mainly limited to a multi-hop scenario where there is no direct link 
between the source and the destination [13]. On the other hand, in [14], Cui et al. consider the so-called receive diversity (RD) protocol of [15] which allows direct link transmission and includes multi-hop scheme as a special case. The focus of [14] is to determine the optimum training symbol sequence and relay precoding structure. Zhang and Gursoy [16] also consider the RD protocol and formulate a training power allocation problem between relay and source nodes based on the maximization of a lower bound on the capacity. In their work, they assume that channel estimation is carried out both at the relay and the destination. As earlier emphasized, this requires that the relay node should be equipped with a channel estimator. A feed-forward control channel should be deployed as well for transmission of the quantized source-torelay channel estimate.

In our work, similar to [14] and [16], we consider RD protocol; however we assume that the relay node is not equipped with a channel estimator to keep its complexity as low as possible. We obtain the channel estimate for cascaded sourcerelay-destination link at the destination node. Considering average received SNR as the objective function and taking into account the effect of relay location, we attempt to answer the following questions:

Q1) How should overall transmit power be shared between training and data transmission periods?

Q2) How should training power be allocated to broadcasting and relaying phases?

Q3) How should data power be allocated to broadcasting and relaying phases?

In our work, we assume two different power allocation constraints which we name as total network power (TNP) and individual node power (INP) constraints. In the first one, it is assumed that the total transmit power can be shared by source and relay nodes. In the second one, source and relay nodes have individual power constraints on the available transmit powers. Therefore, under INP constraint, only Q1 becomes relevant.

For the single-relay AaF cooperative system under consideration, we derive the optimum power allocation rules to maximize the average received SNR at the destination node under either TNP or INP constraints. Optimized schemes are observed to outperform the original schemes with equal power allocation providing SNR gains up to $5.5 \mathrm{~dB}$ depending on the relay location and deployed power constraint.

The rest of the paper is organized as follows: In Section II, we present the channel model and describe the cooperative system under consideration followed by the channel estimation method in Section III. In Sections IV and V, we formulate the power allocation problems, respectively, under TNP and INP constraints and present closed-form analytical solutions for various relay locations. In Section VI, we present a comprehensive Monte-Carlo simulation study and demonstrate the error rate performance of $\mathrm{AaF}$ relaying with optimum power allocation (OPA) and equal power allocation (EPA). Section VII concludes the paper.

Notation: Bold upper-case letters denote the matrices and bold lower-case letters denote the vectors. $(.)^{\mathrm{T}},(.)^{*}$, and $(.)^{\mathrm{H}}$ denote transpose, conjugate, and Hermitian transpose operations respectively. |.| denotes the absolute value and

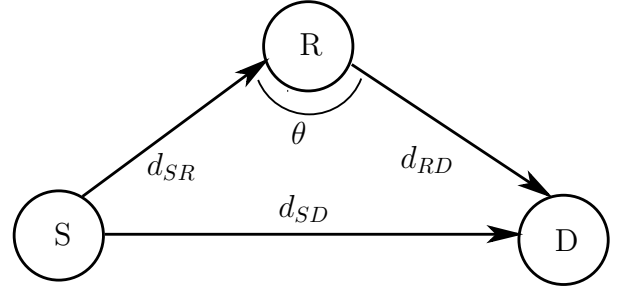

Fig. 1. Relay-assisted transmission model.

$\mathbf{I}_{N}$ denotes the identity matrix of size $N . \mathrm{E}($.$) and \operatorname{var}($.$) ,$ respectively, denote the expectation and the variance of a random variable.

\section{TRAnSmission MOdeL}

We consider a single-relay AaF cooperative network with half-duplex nodes each of which is equipped with a single pair of transmit and receive antennas (Fig. 1). We assume an aggregate channel model which takes into account both longterm propagation effects (i.e., path loss and shadowing) and short-term fading. This lets us explicitly consider the effects of relay location in our transmission model.

In Fig. $1, d_{S D}, d_{S R}$, and $d_{R D}$ are the distances of source-to-destination $(\mathrm{S} \rightarrow \mathrm{D})$, source-to-relay $(\mathrm{S} \rightarrow \mathrm{R})$, and relay-to-destination $(\mathrm{R} \rightarrow \mathrm{D})$ links, respectively, and $\theta$ is the angle between lines $\mathrm{S} \rightarrow \mathrm{R}$ and $\mathrm{R} \rightarrow \mathrm{D}$. Assuming the path loss in $\mathrm{S} \rightarrow \mathrm{D}$ normalized to be unity, the relative geometrical gains of $\mathrm{S} \rightarrow \mathrm{R}$ and $\mathrm{R} \rightarrow \mathrm{D}$ links are defined as $G_{S R}=\left(S_{S D} / S_{S R}\right)\left(d_{S D} / d_{S R}\right)^{\alpha}$ and $G_{R D}=$ $\left(S_{S D} / S_{R D}\right)\left(d_{S D} / d_{R D}\right)^{\alpha}$ where $\alpha$ is the propagation coefficient [17]. Here, $S_{S D}, S_{S R}$, and $S_{R D}$ denote the power loss due to the shadowing for $\mathrm{S} \rightarrow \mathrm{D}, \mathrm{S} \rightarrow \mathrm{R}$, and $\mathrm{R} \rightarrow \mathrm{D}$ links, respectively. The shadowing loss can vary from $6 \mathrm{~dB}$ to $11 \mathrm{~dB}$ according to measurements performed in mobile-to-mobile channels [18]. Relative geometrical gains can be related to each other by $\left(\left(S_{R D} / S_{S D}\right) G_{R D}\right)^{-2 / \alpha}+\left(\left(S_{S R} / S_{S D}\right) G_{S R}\right)^{-2 / \alpha}-$ $2 \cos (\theta)\left(\left(S_{R D} / S_{S D}\right) G_{R D}\right)^{-1 / \alpha}\left(\left(S_{S R} / S_{S D}\right) G_{S R}\right)^{-1 / \alpha}=$ 1 through cosine theorem. We can further define the ratio of relative geometrical gains as $\beta=G_{S R} / G_{R D}=$ $\left(S_{R D} / S_{S R}\right)\left(d_{R D} / d_{S R}\right)^{\alpha}$ which indicates the location of the relay with respect to the source and the destination. The more negative this ratio (given in $\mathrm{dB}$ ) is, the closer the relay is placed to the destination. On the other hand, the more positive this ratio (given in $\mathrm{dB}$ ) is, the closer the relay is placed to the source.

The transmission model under consideration builds upon RD cooperation protocol [15]. This protocol effectively implements a SIMO (single-input multiple-output) scheme in a distributed fashion realizing receive diversity advantages. In $\mathrm{RD}$ protocol, the source node communicates with the relay and the destination over the first time slot (i.e., broadcasting phase). In the second time slot (i.e., relaying phase), only the relay node communicates with the destination. Therefore, the signal transmitted to both the relay and the destination nodes over two time slots is the same.

We consider coherent detection with pilot-assisted channel estimation which relies on the insertion of known training (pilot) symbols in information-bearing data [19], [20]. These 
pilot symbols and the specific multiplexing scheme are known at the receiver and used for channel estimation purpose. Since we consider a quasi-static fading channel, the placement of pilot symbols is irrelevant in our optimization. In the following, we introduce the received signal models under TNP and INP constraints.

\section{A. TNP Constraint}

In our work, we consider a frame length of $T=T_{t}+T_{d}$ symbols where $T_{t}$ and $T_{d}$ denote the length of training and data symbols, respectively. Let $2 P T$ be the total available energy consumed during the transmission of $T$ symbols, yielding an average power of $P$ (per time slot). Over the two time slots (required to transmit a symbol for the cooperation protocol under consideration), $2 P_{t}$ and $2 P_{d}$ are, respectively, assigned for the transmission of a training symbol and a data symbol. Therefore, we have $2 P_{t} T_{t}+2 P_{d} T_{d}=2 P T$. We further introduce a parameter $0<\rho<1$ to relate $P_{d}$ and $P_{t}$ as $P_{d}=\rho P T / T_{d}$ and $P_{t}=(1-\rho) P T / T_{t}$. This parameter (relevant to $\mathrm{Q} 1$ ) will be later used in the optimization procedure to determine how much of the total power should be allocated to data or training symbol transmission.

Let $x_{j}$ denote M-PSK (phase shift keying) modulation symbol with normalized power $\mathrm{E}\left[\left|x_{j}\right|^{2}\right]=1$ for $j=1,2, \ldots ., T_{d}$. The received signals at the relay and the destination are given by

$$
\begin{gathered}
r_{1, j}=\sqrt{2 K_{d} G_{S R} P_{d}} h_{S R} x_{j}+n_{1, j} \\
r_{2, j}=\sqrt{2 K_{d} P_{d}} h_{S D} x_{j}+n_{2, j}
\end{gathered}
$$

where $0<K_{d}<1$ is defined as another optimization parameter (relevant to Q3) which controls the fraction of power reserved for the source node's use in the broadcasting phase of data transmission period. The relay node normalizes the received signal by a factor of $\sqrt{\mathrm{E}\left[\left|r_{1, j}\right|^{2}\right]}$ to have average unit energy and then forwards the scaled signal with power $2\left(1-K_{d}\right) P_{d}$ during the relaying phase. The received signal at the destination is, therefore, given by

$$
r_{3, j}=\sqrt{2\left(1-K_{d}\right) G_{R D} P_{d}} h_{R D} \frac{r_{1, j}}{\sqrt{\mathrm{E}\left[\left|r_{1, j}\right|^{2}\right]}}+n_{3, j} .
$$

In (1)-(3), $n_{1, j}, n_{2, j}$, and $n_{3, j}$ model the additive noise terms and are the independent samples of zero-mean complex Gaussian random variables with variance of $N_{0} / 2$ per dimension. $h_{S R}, h_{S D}$, and $h_{R D}$ denote the Rayleigh fading coefficients over $\mathrm{S} \rightarrow \mathrm{R}, \mathrm{S} \rightarrow \mathrm{D}$, and $\mathrm{R} \rightarrow \mathrm{D}$ links and are modeled as zero-mean complex Gaussian fading coefficients with variance of 0.5 per dimension. Replacing the normalization factor $\sqrt{\mathrm{E}\left[\left|r_{1, j}\right|^{2}\right]}=\sqrt{2 K_{d} G_{S R} P_{d}+N_{0}}$ in (3), we have

$$
r_{3, j}=\sqrt{\frac{4 K_{d}\left(1-K_{d}\right) G_{S R} G_{R D} P_{d}^{2}}{2 K_{d} G_{S R} P_{d}+N_{0}}} h_{S R} h_{R D} x_{j}+\tilde{n}
$$

where $\tilde{n}=\sqrt{\left[2\left(1-K_{d}\right) G_{R D} P_{d}\right] /\left(2 K_{d} G_{S R} P_{d}+N_{0}\right)} h_{R D} n_{1, j}$ $+n_{3, j}$. Due to the term involving $h_{R D} n_{1, j}, \quad \tilde{n}$ is of nonGaussian nature which makes the analysis intractable for most cases. However, as in [10], [21], we can treat it as Gaussian noise. Such an approximation results in a pessimistic performance estimate, thereby upper bounding the original performance, but slope of the curve on a log-log scale (which determines the diversity order) remains the same. After replacing $\tilde{n}$ with zeromean Gaussian noise with the same average power, the destination node normalizes the received signal by a factor of $\sqrt{1+2\left(1-K_{d}\right) G_{R D} P_{d} /\left(2 K_{d} G_{S R} P_{d}+N_{0}\right)}$ which yields ${ }^{1}$

$$
r_{4, j}=\sqrt{B_{d} P_{d}} h_{S R} h_{R D} x_{j}+n_{4, j}
$$

where $n_{4, j}$ is zero-mean complex Gaussian random variable with variance of $N_{0}$ and $B_{d}$ is given by

$$
B_{d}=\frac{4 K_{d}\left(1-K_{d}\right) G_{S R} G_{R D} P_{d} / N_{0}}{1+2 K_{d} G_{S R} P_{d} / N_{0}+2\left(1-K_{d}\right) G_{R D} P_{d} / N_{0}}
$$

Introducing $\quad \mathbf{r}_{d, j}=\left[\begin{array}{ll}r_{2, j} & r_{4, j}\end{array}\right]^{T}$ and $\mathbf{n}_{d, j}=$ $\left[\begin{array}{ll}n_{2, j} & n_{4, j}\end{array}\right]^{T}$, the received signals for the $j^{t h}$ data block can be given as $\mathbf{r}_{d, j}=\mathbf{X}_{d, j} \mathbf{h}+\mathbf{n}_{d, j}$ where $\mathbf{h}=\left[\begin{array}{ll}h_{S R} h_{R D} & h_{S D}\end{array}\right]^{T}$ and $\mathbf{X}_{d, j}$ is

$$
\mathbf{X}_{d, j}=\left[\begin{array}{cc}
0 & \sqrt{2 K_{d} P_{d}} \\
\sqrt{B_{d} P_{d}} & 0
\end{array}\right] x_{j} .
$$

The received signals $\mathbf{r}_{d, j}$ during the data transmission period can be then stacked and written in a compact matrix form as

$$
\mathbf{r}_{d}=\left[\mathbf{r}_{d, 1}, \mathbf{r}_{d, 2}, \ldots \mathbf{r}_{d, T_{d}}\right]^{\mathrm{T}}=\mathbf{X}_{d} \mathbf{h}+\mathbf{n}_{d}
$$
where $\quad \mathbf{X}_{d}=\left[\begin{array}{llll}\mathbf{X}_{d, 1} & \mathbf{X}_{d, 2} & \cdots & \mathbf{X}_{d, T_{d}}\end{array}\right]^{\mathrm{T}}$ and $\mathbf{n}_{d}=$
$\left[\begin{array}{lll}\mathbf{n}_{d, 1} \mathbf{n}_{d, 2} & \cdots \mathbf{n}_{d, T_{d}}\end{array}\right]^{\mathrm{T}}$.

\section{B. INP Constraint}

Unlike TNP constraint where relay and source nodes are allowed to share the total transmit power, we now consider the case where relay and source nodes have individual power budgets. Therefore, under INP constraint, only Q1 becomes relevant. Let $P_{S}$ and $P_{R}$ denote the source and the relay powers. We introduce parameters $0<\rho_{S}, \rho_{R}<1$ which are used to determine how much of the individual power should be allocated to data or training symbol transmission for each node. For the source node, the power allocated to data transmission is given by $P_{d, S}=\rho_{S} P_{S} T / T_{d}$, while training power is given by $P_{t, S}=\left(1-\rho_{S}\right) P_{S} T / T_{t}$. In a similar manner, for the relay node, we can write $P_{d, R}=\rho_{R} P_{R} T / T_{d}$ and $P_{t, R}=\left(1-\rho_{R}\right) P_{R} T / T_{t}$. Following similar steps in the previous section, it can be shown that the received signals can be written in the form of (8) where $\mathbf{X}_{d, j}$ and $B_{d}$ are now replaced by

$$
\begin{gathered}
\mathbf{X}_{d, j}=\left[\begin{array}{cc}
0 & \sqrt{P_{d, S}} \\
\sqrt{B_{d} P_{d, R}} & 0
\end{array}\right] x_{j}, \\
B_{d}=\frac{G_{R D} G_{S R} P_{d, S} / N_{0}}{G_{R D} P_{d, R} / N_{0}+G_{S R} P_{d, S} / N_{0}+1} .
\end{gathered}
$$

\footnotetext{
${ }^{1}$ This does not change the signal-to-noise ratio, but simplifies the ensuing presentation [3].
} 


\section{Channel Estimation and Decoding}

During the training period, prior to data transmission, the source node transmits pilot symbols for channel estimation purpose. The training matrix $\mathbf{X}_{t, i}$ for $i=1,2, \ldots T_{t}$ has a similar form of (7)-(10). Under TNP constraint, it is given by

$$
\mathbf{X}_{t, i}=\left[\begin{array}{cc}
0 & \sqrt{2 K_{t} P_{t}} \\
\sqrt{B_{t} P_{t}} & 0
\end{array}\right] p
$$

where $p$ denotes the pilot symbol and $B_{t}$ is defined as

$$
B_{t}=\frac{4 K_{t}\left(1-K_{t}\right) G_{S R} G_{R D} P_{t} / N_{0}}{1+2 K_{t} G_{S R} P_{t} / N_{0}+2\left(1-K_{t}\right) G_{R D} P_{t} / N_{0}}
$$

where we introduce another optimization parameter $0<$ $K_{t}<1$ (relevant to Q2) which controls the allocation of training power to broadcasting and relaying phases. Under INP constraint, training matrix $\mathbf{X}_{t, i}$ is given by

$$
\mathbf{X}_{t, i}=\left[\begin{array}{cc}
0 & \sqrt{P_{t, S}} \\
\sqrt{B_{t} P_{t, R}} & 0
\end{array}\right] x_{i}
$$

with

$$
B_{t}=\frac{G_{R D} G_{S R} P_{t, S} / N_{0}}{G_{R D} P_{t, R} / N_{0}+G_{S R} P_{t, S} / N_{0}+1} .
$$

The received signals during the training period are given by $\mathbf{r}_{t}=\mathbf{X}_{t} \mathbf{h}+\mathbf{n}_{t}$ where $\mathbf{X}_{t}=\left[\mathbf{X}_{t, 1} \mathbf{X}_{t, 2} \cdots \mathbf{X}_{t, T_{t}}\right]^{\mathrm{T}}$ and $\mathbf{n}_{t}=$ $\left[\mathbf{n}_{t, 1} \mathbf{n}_{t, 2} \cdots \mathbf{n}_{t, T_{t}}\right]^{\mathrm{T}}$. Based on an LMMSE (Linear Minimum Mean Squared Error) estimator, estimate of the channel vector is obtained as $\hat{\mathbf{h}}=\mathbf{B} \mathbf{r}_{t}$ where $\mathbf{B}=\mathrm{E}\left(\mathbf{h} \mathbf{r}_{t}^{\mathrm{H}}\right) \mathrm{E}\left(\mathbf{r}_{t} \mathbf{r}_{t}^{\mathrm{H}}\right)^{-1}$ [22]. After some mathematical manipulations, $\hat{\mathbf{h}}$ can be written as

$$
\hat{\mathbf{h}}=\left[\begin{array}{ll}
\hat{h}_{S R D} & \hat{h}_{S D}
\end{array}\right]^{\mathrm{T}}=\mathbf{X}_{t}^{\mathrm{H}}\left(\mathbf{X}_{t} \mathbf{X}_{t}^{\mathrm{H}}+N_{0} \mathbf{I}_{2}\right)^{-1} \mathbf{r}_{t} .
$$

Let $\mathbf{e}=\mathbf{h}-\hat{\mathbf{h}}$ denote the estimation error. Its covariance matrix is given by

$$
\begin{aligned}
\mathbf{C}_{\mathbf{e}} & =\mathrm{E}\left(\mathbf{h h}^{\mathrm{H}}\right)-\mathbf{B E}\left(\mathbf{r}_{t} \mathbf{r}_{t}^{\mathrm{H}}\right) \mathbf{B}^{\mathrm{H}} \\
& =N_{0}\left(\mathbf{X}_{t}^{\mathrm{H}} \mathbf{X}_{t}+N_{0} \mathrm{I}_{2}\right)^{-1} .
\end{aligned}
$$

Eq. (16) has a diagonal structure and its diagonal elements $\sigma_{e_{S R D}}^{2}$ and $\sigma_{e_{S D}}^{2}$ are given by

$$
\begin{aligned}
& \operatorname{var}\left(e_{S R D}\right)=\sigma_{e_{S R D}}^{2}=N_{0} /\left(B_{t} T_{t} P_{t}+N_{0}\right), \\
& \operatorname{var}\left(e_{S D}\right)=\sigma_{e_{S D}}^{2}=N_{0} /\left(2 K_{t} T_{t} P_{t}+N_{0}\right) .
\end{aligned}
$$

During the data transmission period, the received signals along with channel estimate vector are fed to a maximum likelihood (ML) decoder which is given by

$$
\arg \min _{\mathbf{X}_{d}}\left\|\mathbf{r}_{d}-\mathbf{X}_{d} \hat{\mathbf{h}}\right\|^{2}
$$

as if the channels are perfectly known. This results in so-called mismatched-coherent decoding [23].
TABLE I

OPTIMIZATION PARAMETERS UNDER TNP AND INP CONSTRAINTS

\begin{tabular}{|c||c|c|}
\hline & TNP & INP \\
\hline & & $P_{d, R}=\rho_{R} P_{R} T / T_{d}$ \\
Q1 & $P_{d}=\rho P T / T_{d}$ & $P_{d, S}=\rho_{S} P_{S} T / T_{d}$ \\
& $P_{t}=(1-\rho) P T / T_{t}$ & $\begin{array}{c}P_{t, R}=\rho_{R} P_{R} T / T_{t} \\
P_{t, S}=\rho_{S} P_{S} T / T_{t}\end{array}$ \\
\hline $\mathbf{Q 2}$ & $\begin{array}{c}2 K_{t} P_{t} \\
2\left(1-K_{t}\right) P_{t}\end{array}$ & N/A \\
\hline $\mathbf{Q 3}$ & $\begin{array}{c}2 K_{d} P_{d} \\
2\left(1-K_{d}\right) P_{d}\end{array}$ & N/A \\
\hline
\end{tabular}

\section{OPTIMIZATION OF $K_{d}, K_{t}$ AND $\rho$ UNDER TNP CONSTRAINT}

Under TNP constraint, we have introduced three optimization parameters, namely $K_{d}, K_{t}$, and $\rho$ where $\rho$ controls the power allocation to training and data periods. On the other hand, $K_{d}$ and $K_{t}$ respectively control the allocation of data and training powers between broadcasting and relaying phases. Therefore, we can find the answers to our previously posed three questions as summarized in Table I. Here, we aim to optimize these parameters in order to maximize the average received SNR at the destination node. The average SNR at the destination is

$$
\begin{array}{r}
S N R_{\text {avg }}=S N R_{S \rightarrow D}+S N R_{S \rightarrow R \rightarrow D} \\
=\frac{2 K_{d} P_{d} \operatorname{var}\left(\hat{h}_{S D}\right)}{2 K_{d} P_{d} \operatorname{var}\left(e_{S D}\right)+N_{0}}+\frac{B_{d} P_{d} \operatorname{var}\left(\hat{h}_{S R D}\right)}{B_{d} P_{d} \operatorname{var}\left(e_{S R D}\right)+N_{0}}
\end{array}
$$

where $\operatorname{var}\left(\hat{h}_{S D}\right)$ and $\operatorname{var}\left(\hat{h}_{S D}\right)$ denote the variances of $\mathrm{S} \rightarrow \mathrm{D}$ and $\mathrm{S} \rightarrow \mathrm{R} \rightarrow \mathrm{D}$ channel estimates and are given by

$$
\begin{gathered}
\operatorname{var}\left(\hat{h}_{S D}\right)=2 K_{t} T_{t} P_{t} /\left(2 K_{t} T_{t} P_{t}+N_{0}\right), \\
\operatorname{var}\left(\hat{h}_{S R D}\right)=B_{t} T_{t} P_{t} /\left(B_{t} T_{t} P_{t}+N_{0}\right) .
\end{gathered}
$$

After rewriting the $S N R_{S \rightarrow D}$ and $S N R_{S \rightarrow R \rightarrow D}$ expressions of (20) in common denominator, we can formulate our optimization problem as

$$
\begin{gathered}
\max _{\rho, K_{t}, K_{d}} \Phi_{T N P}\left(\rho, K_{t}, K_{d}\right) \\
\text { s.t. } 0<\rho<1,0<K_{t}<1 \text { and } 0<K_{d}<1
\end{gathered}
$$

where the objective function $\Phi_{T N P}\left(\rho, K_{t}, K_{d}\right)$ is

$$
\begin{aligned}
\Phi_{T N P}= & \frac{2 K_{d} P_{d} \operatorname{var}\left(\hat{h}_{S D}\right) / N_{0}}{\left(2 K_{d} P_{d} \operatorname{var}\left(e_{S D}\right) / N_{0}+1\right)} \\
& +\frac{B_{d} P_{d} \operatorname{var}\left(\hat{h}_{S R D}\right) / N_{0}}{\left(B_{d} P_{d} \operatorname{var}\left(e_{S R D}\right) N_{0}+1\right)} .
\end{aligned}
$$

Inserting (17), (18), (21) and (22) in (24) and further replacing $P_{d}=\rho P T / T_{d}$ and $P_{t}=(1-\rho) P T / T_{t}$ in the resulting expression, we obtain

$$
\begin{aligned}
\Phi_{T N P} & =\frac{4 K_{d} K_{t} \rho(1-\rho)\left(P T / N_{0}\right)^{2} / T_{d}}{\left(2 K_{d} \rho P T / T_{d} N_{0}+2 K_{t}(1-\rho) P T / N_{0}+1\right)} \\
& +\frac{B_{d} B_{t} \rho(1-\rho)\left(P T / N_{0}\right)^{2} / T_{d}}{\left(B_{d} \rho P T / T_{d} N_{0}+B_{t} 2 K_{t}(1-\rho) P T / N_{0}+1\right)}
\end{aligned}
$$


where $B_{d}$ and $B_{t}$, earlier defined by (6) and (12), are functions of optimization parameters, and can be expanded as

$$
\begin{gathered}
B_{d}=\frac{4 K_{d}\left(1-K_{d}\right) G_{S R} G_{R D} \frac{\rho P T}{T_{d} N_{0}}}{1+2 K_{d} G_{S R} \frac{\rho P T}{T_{d} N_{0}}+2\left(1-K_{d}\right) G_{R D} \frac{\rho P T}{T_{d} N_{0}}}, \\
B_{t}=\frac{4 K_{t}\left(1-K_{t}\right) G_{S R} G_{R D} \frac{(1-\rho) P T}{T_{t} N_{0}}}{1+2 K_{t} G_{S R} \frac{(1-\rho) P T}{T_{t} N_{0}}+2\left(1-K_{t}\right) G_{R D} \frac{(1-\rho) P T}{T_{t} N_{0}}} .
\end{gathered}
$$

A general analytic solution for optimization parameters is very difficult, if not impossible. Therefore, in the following, we investigate representative scenarios in which (25) simplifies and lets us provide closed-form solutions.

\section{A. Scenario I: Relay is Close to Destination}

When the relay is located close to the destination node, we have $\beta<<1$ which lets us write $G_{S R} \approx 1$ and $G_{R D}>>$ 1. Therefore, (26) and (27) simplify as $B_{d} \approx 2 K_{d} G_{S R} \approx$ $2 K_{d}$ and $B_{t} \approx 2 K_{t} G_{S R} \approx 2 K_{t}$. Inserting these in (25), the objective function is formulated as

$$
\Phi_{T N P, I}=\frac{8 K_{d} K_{t} \frac{\rho(1-\rho)\left(P T / N_{0}\right)^{2}}{T_{d}}}{\left(2 K_{d} \frac{\rho P T}{T_{d} N_{0}}+2 K_{t} \frac{(1-\rho) P T}{N_{0}}+1\right)} .
$$

For a fixed value of $\rho$, it can be easily checked that (28) is an increasing function of $K_{d}$ and $K_{t}$ (i.e., both $d \Phi_{T, D} / d K_{d}$ and $d \Phi_{T, D} / d K_{t}$ are greater than zero). Therefore, it can be maximized by setting $K_{d}$ and $K_{t}$ as large as possible. Considering the ranges of $K_{d}$ and $K_{t}$, i.e., $0<K_{d}, K_{t}<1$, we conclude that the objective function is maximized for $K_{d_{-} o p t} \rightarrow 1$ and $K_{t_{-} o p t} \rightarrow 1$. This points out that majority of the training/data power should be allocated for the use of source node in the broadcasting phase.

Differentiating (28) with respect to $\rho$, inserting $K_{d_{-} \text {opt }}$ and $K_{t_{-} o p t}$ in the resulting expression, and equating it to zero, optimum value of $\rho$ is found as

$$
\rho_{\text {opt }}=\left\{\begin{array}{ll}
1 / 2 & \text { for } \quad T_{d}=1 \\
\frac{T_{d}\left(N_{0}+2 K_{t_{-} o p t} P T\right)}{2 P T\left(T_{d} K_{t_{-} o p t}-K_{d_{-} o p t}\right)} & \text { for } \quad T_{d}>1 \\
\times\left(1-\sqrt{1-\frac{2 P T\left(T_{d} K_{t_{-} o p t}-K_{d_{-} o p t}\right)}{T_{d}\left(N_{0}+2 K_{t_{-} o p t} P T\right)}}\right)
\end{array} .\right.
$$

Table II tabulates optimum values of $\rho$ for a frame length of $T=100$ assuming various number of pilot symbols, i.e., $T_{t}=1,5,20,50,80$ and $\beta=-30 \mathrm{~dB}$. For $T_{t}=1$, we observe that the optimum $\rho$ value is $\sim 0.9^{2}$. This indicates that allocating $10 \%$ of the total power to training phase would be sufficient to optimize the performance. As $T_{t}$ increases, optimum $\rho$ value, along with number of data symbols (i.e., $T_{d}=T-T_{t}$ ) slightly decreases. This leads to higher $P_{t} T_{t}=\left(1-\rho_{\text {opt }}\right) P T$ values. From (17) and (18), we can readily check that channel estimation errors are inversely proportional to $P_{t} T_{t}$. Therefore, the increase in

\footnotetext{
${ }^{2}$ We have also found optimum values of $\rho$ for various frame lengths (excluded from the table due to space limitations). They give very similar results. For example $\rho_{\text {opt }}$ is 0.87 and 0.89 for $T=50$ and 80 , respectively.
}

\begin{tabular}{|c|c|c|c|c|c|}
\hline$P / N_{0}$ & $T_{t}=1$ & $T_{t}=5$ & $T_{t}=20$ & $T_{t}=50$ & $T_{t}=80$ \\
\hline \multicolumn{6}{|c|}{ Scenario 1: Relay is close to destination $(\beta=-30 \mathrm{~dB})$} \\
\hline $0 \mathrm{~dB}$ & $\begin{array}{c}0.8908 \\
(0.8913)\end{array}$ & $\begin{array}{c}0.8894 \\
(0.8896)\end{array}$ & $\begin{array}{c}0.8834 \\
(0.8825)\end{array}$ & $\begin{array}{c}0.8638 \\
(0.8612)\end{array}$ & $\begin{array}{c}0.8104 \\
(0.8063)\end{array}$ \\
\hline $5 \mathrm{~dB}$ & $\begin{array}{c}0.9025 \\
(0.9027)\end{array}$ & $\begin{array}{c}0.9009 \\
(0.9010)\end{array}$ & $\begin{array}{c}0.8940 \\
(0.8936)\end{array}$ & $\begin{array}{c}0.8720 \\
(0.8709)\end{array}$ & $\begin{array}{c}0.8150 \\
(0.8134)\end{array}$ \\
\hline $10 \mathrm{~dB}$ & $\begin{array}{c}0.9067 \\
(0.9067)\end{array}$ & $\begin{array}{c}0.9050 \\
(0.9050)\end{array}$ & $\begin{array}{c}0.8977 \\
(0.8976)\end{array}$ & $\begin{array}{c}0.8748 \\
(0.8744)\end{array}$ & $\begin{array}{c}0.8165 \\
(0.8160)\end{array}$ \\
\hline $15 \mathrm{~dB}$ & $\begin{array}{c}0.9080 \\
(0.9081)\end{array}$ & $\begin{array}{c}0.9063 \\
(0.9063)\end{array}$ & $\begin{array}{c}0.8989 \\
(0.8988)\end{array}$ & $\begin{array}{c}0.8757 \\
(0.8756)\end{array}$ & $\begin{array}{c}0.8170 \\
(0.8168)\end{array}$ \\
\hline $20 \mathrm{~dB}$ & $\begin{array}{c}0.9085 \\
(0.9085)\end{array}$ & $\begin{array}{c}0.9068 \\
(0.9068)\end{array}$ & $\begin{array}{c}0.8993 \\
(0.8992)\end{array}$ & $\begin{array}{c}0.8760 \\
(0.8760)\end{array}$ & $\begin{array}{c}0.8172 \\
(0.8171)\end{array}$ \\
\hline $25 \mathrm{~dB}$ & $\begin{array}{c}0.9086 \\
(0.9086)\end{array}$ & $\begin{array}{c}0.9069 \\
(0.9069)\end{array}$ & $\begin{array}{c}0.8994 \\
(0.8994)\end{array}$ & $\begin{array}{c}0.8761 \\
(0.8760)\end{array}$ & $\begin{array}{c}0.8172 \\
(0.8172)\end{array}$ \\
\hline \multicolumn{6}{|c|}{ Scenario 2: Relay is close to source $(\beta=30 \mathrm{~dB})$} \\
\hline $0 \mathrm{~dB}$ & $\begin{array}{c}0.8534 \\
(0.8828)\end{array}$ & $\begin{array}{c}0.8525 \\
(0.8814)\end{array}$ & $\begin{array}{c}0.8485 \\
(0.8753)\end{array}$ & $\begin{array}{c}0.8347 \\
(0.8558)\end{array}$ & $\begin{array}{c}0.7924 \\
(0.8038)\end{array}$ \\
\hline $5 \mathrm{~dB}$ & $\begin{array}{c}0.8867 \\
(0.8984)\end{array}$ & $\begin{array}{c}0.8854 \\
(0.8968)\end{array}$ & $\begin{array}{c}0.8797 \\
(0.8900)\end{array}$ & $\begin{array}{c}0.8608 \\
(0.8685)\end{array}$ & $\begin{array}{c}0.8087 \\
(0.8126)\end{array}$ \\
\hline $10 \mathrm{~dB}$ & $\begin{array}{c}0.9010 \\
(0.9051)\end{array}$ & $\begin{array}{c}0.8994 \\
(0.9034)\end{array}$ & $\begin{array}{c}0.8927 \\
(0.8962)\end{array}$ & $\begin{array}{c}0.8709 \\
(0.8735)\end{array}$ & $\begin{array}{c}0.8145 \\
(0.8157)\end{array}$ \\
\hline $15 \mathrm{~dB}$ & $\begin{array}{c}0.9062 \\
(0.9075)\end{array}$ & $\begin{array}{c}0.9045 \\
(0.9058)\end{array}$ & $\begin{array}{c}0.8972 \\
(0.8984)\end{array}$ & $\begin{array}{c}0.8744 \\
(0.8753)\end{array}$ & $\begin{array}{c}0.8164 \\
(0.8168)\end{array}$ \\
\hline $20 \mathrm{~dB}$ & $\begin{array}{c}0.9079 \\
(0.9083)\end{array}$ & $\begin{array}{c}0.9062 \\
(0.9067)\end{array}$ & $\begin{array}{c}0.8987 \\
(0.8991)\end{array}$ & $\begin{array}{c}0.8756 \\
(0.8758)\end{array}$ & $\begin{array}{c}0.8170 \\
(0.8171)\end{array}$ \\
\hline $25 \mathrm{~dB}$ & $\begin{array}{c}0.9084 \\
(0.9086)\end{array}$ & $\begin{array}{c}0.9067 \\
(0.9068)\end{array}$ & $\begin{array}{c}0.8992 \\
(0.8993)\end{array}$ & $\begin{array}{c}0.8759 \\
(0.8760)\end{array}$ & $\begin{array}{c}0.8172 \\
(0.8172)\end{array}$ \\
\hline \multicolumn{6}{|c|}{ Scenario 3: Relay is in the middle $(\beta=0 \mathrm{~dB})$} \\
\hline $0 \mathrm{~dB}$ & $\begin{array}{c}0.9087 \\
(0.8914)\end{array}$ & $\begin{array}{c}0.9069 \\
(0.8877)\end{array}$ & $\begin{array}{c}0.8994 \\
(0.8743)\end{array}$ & $\begin{array}{c}0.8761 \\
(0.8427)\end{array}$ & $\begin{array}{c}0.8173 \\
(0.7788)\end{array}$ \\
\hline $5 \mathrm{~dB}$ & $\begin{array}{c}0.9087 \\
(0.9027)\end{array}$ & $\begin{array}{c}0.9069 \\
(0.9003)\end{array}$ & $\begin{array}{c}0.8994 \\
(0.8905)\end{array}$ & $\begin{array}{c}0.8761 \\
(0.8635)\end{array}$ & $\begin{array}{c}0.8173 \\
(0.8022)\end{array}$ \\
\hline $10 \mathrm{~dB}$ & $\begin{array}{c}0.9087 \\
(0.9067)\end{array}$ & $\begin{array}{c}0.9069 \\
(0.9048)\end{array}$ & $\begin{array}{c}0.8994 \\
(0.8965)\end{array}$ & $\begin{array}{c}0.8761 \\
(0.8718)\end{array}$ & $\begin{array}{c}0.8173 \\
(0.8120)\end{array}$ \\
\hline $15 \mathrm{~dB}$ & $\begin{array}{c}0.9087 \\
(0.9080)\end{array}$ & $\begin{array}{c}0.9069 \\
(0.9063)\end{array}$ & $\begin{array}{c}0.8994 \\
(0.8985)\end{array}$ & $\begin{array}{c}0.8761 \\
(0.8747)\end{array}$ & $\begin{array}{c}0.8173 \\
(0.8155)\end{array}$ \\
\hline $20 \mathrm{~dB}$ & $\begin{array}{c}0.9087 \\
(0.9085)\end{array}$ & $\begin{array}{c}0.9069 \\
(0.9067)\end{array}$ & $\begin{array}{c}0.8994 \\
(0.8991)\end{array}$ & $\begin{array}{c}0.8761 \\
(0.8757)\end{array}$ & $\begin{array}{c}0.8173 \\
(0.8167)\end{array}$ \\
\hline $25 \mathrm{~dB}$ & $\begin{array}{c}0.9087 \\
(0.9086)\end{array}$ & $\begin{array}{c}0.9069 \\
(0.9069)\end{array}$ & $\begin{array}{c}0.8994 \\
(0.8993)\end{array}$ & $\begin{array}{c}0.8761 \\
(0.8760)\end{array}$ & $\begin{array}{c}0.8173 \\
(0.8171)\end{array}$ \\
\hline
\end{tabular}

TABLE II

OPTIMUM $\rho$ VALUES FOR VARIOUS $T$ VALUES UNDER TNP CONSTRAINT $\left(T=100, \quad \theta=\pi, \quad \alpha=2, \quad S_{S R}=S_{R D}\right)$.

Numbers in parenthesis are obtained through numerical optimization of exact objective function given by (25).

training interval would be expected to result in a better channel estimation quality and less bit errors at the decoder. However, this comes with a sacrifice in data throughput and channel capacity. In Table II, we have further included optimization values obtained through numerical optimization based on the exact form of objective function. Comparison of those with the analytical results shows a very good match in general and identical results in many occasions.

\section{B. Scenario II: Relay is Close to Source}

When the relay is located near the source node, the relative gain ratio becomes $\beta>>1$ which leads to $G_{R D} \approx 1$ and 
$G_{S R}>>1$. Consequently, we have $B_{d} \approx 2 G_{R D}\left(1-K_{d}\right) \approx$ $2-2 K_{d}$ and $B_{t} \approx 2 G_{R D}\left(1-K_{t}\right) \approx 2-2 K_{t}$. Inserting these into (25), we have

$$
\begin{aligned}
& \Phi_{T N P, I I}=\frac{4 K_{d} K_{t} \rho(1-\rho) \frac{\left(P T / N_{0}\right)^{2}}{T_{d}}}{\left(2 K_{d} \frac{\rho P T}{T_{d} N_{0}}+2 K_{t} \frac{(1-\rho) P T}{N_{0}}+1\right)}+ \\
& \frac{\left(2-2 K_{d}\right)\left(2-2 K_{t}\right) \rho(1-\rho) \frac{\left(P T / N_{0}\right)^{2}}{T_{d}}}{\left(\left(2-2 K_{d}\right) \frac{\rho P T}{T_{d} N_{0}}+\left(2-2 K_{t}\right) \frac{(1-\rho) P T}{N_{0}}+1\right)} .
\end{aligned}
$$

There is no easy way of solving (30) analytically for optimum values. However, under the assumption of $K_{t}=K_{d}$ which would be valid under sufficiently high SNR values (see Appendix), (30) reduces to (31) given at the top of next page. Under the high SNR assumption, we can further safely ignore the terms with $\left(P T / N_{0}\right)^{2}$ in numerator and second term in denominator of (31). After some simplifications, (31) yields

$$
\Phi_{T N P, I I}=\frac{8 K_{d}\left(1-K_{d}\right) \frac{\rho(1-\rho)\left(P T / N_{0}\right)^{2}}{T_{d}}}{4 K_{d}\left(1-K_{d}\right)\left(\frac{\rho P T}{T_{d} N_{0}}+(1-\rho) \frac{P T}{N_{0}}\right)+2} .
$$

Eq. (32) is an increasing function of $K_{d}\left(1-K_{d}\right)$ for a fixed $\rho$ value. Maximum value of $K_{d}\left(1-K_{d}\right)$ takes place at $K_{d_{-} o p t}=K_{t_{-} o p t}=0.5$. After differentiating (32) and equating the resulting expression to zero, we obtain the optimum $\rho$ in terms of $K_{d_{-} \text {opt }}$ as

$$
\rho_{\text {opt }}= \begin{cases}1 / 2 & \text { for } T_{d}=1 \\ \frac{1+2 K_{d_{-} o p t}\left(1-K_{d_{-} o p t}\right) \frac{P T}{N_{0}}}{2 K_{d_{-} o p t}\left(1-K_{d_{-} o p t}\right) \frac{P T}{N_{0}}\left(1-\frac{1}{T_{d}}\right)} & \text { for } T_{d}>1 \\ \times\left(1-\sqrt{\left.1-\frac{2 K_{d_{-} o p t}\left(1-K_{d_{\_} o p t}\right) \frac{P T}{N_{0}}\left(1-\frac{1}{T_{d}}\right)}{1+2 K_{d_{-} o p t}\left(1-K_{d_{-} o p t}\right) \frac{P T}{N_{0}}}\right)}\right.\end{cases}
$$

Table II tabulates optimum values of $\rho$ for a frame length of $T=100$ assuming various number of pilot symbols and $\beta=$ $30 \mathrm{~dB}$. Specifically for $T_{t}=1, \rho_{\text {opt }}$ is found $\sim 0.9$. Similar to the previous scenario, we observe that optimum $\rho$ value decreases as the training length increases. In contrast, $K_{d_{-} o p t}$ and $K_{t_{-} o p t}$ drop from 1 to 0.5 . This indicates that the training power allocated to the relay node should increase as the relay moves closer to source node and under such scenarios relay and source nodes should share the training power equally.

\section{Scenario III: Relay is in the Midway between Source and Destination}

When the relay is equidistant from the source and destination nodes (along with the assumption that $\mathrm{S} \rightarrow \mathrm{R}$ and $\mathrm{R} \rightarrow \mathrm{D}$ links experience the same shadowing effect, i.e., $S_{R D}=S_{S R}$ ), the relative channel gain ratio becomes $\beta=1$. Therefore, we have $G_{S R}=G_{R D}$; simplifying (26) and (27) to $B_{d} \approx$ $2 K_{d}\left(1-K_{d}\right) G_{S R}$ and $B_{t} \approx 2 K_{t}\left(1-K_{t}\right) G_{S R}$. Inserting these in (25), we have

$$
\begin{gathered}
\Phi_{T N P, I I I}=\frac{4 K_{d} K_{t} \rho(1-\rho) \frac{\left(P T / N_{0}\right)^{2}}{T_{d}}}{\left(2 K_{d} \frac{\rho P T}{T_{d} N_{0}}+2 K_{t} \frac{(1-\rho) P T}{N_{0}}+1\right)}+ \\
\frac{4 K_{t} K_{d}\left(1-K_{t}\right)\left(1-K_{d}\right) G_{S R}^{2} \rho(1-\rho) \frac{\left(P T / N_{0}\right)^{2}}{T_{d}}}{\left(2 G_{S R} \frac{P T}{N_{0}}\left(K_{d}\left(1-K_{d}\right) \frac{\rho}{T_{d}}+K_{t}\left(1-K_{t}\right)(1-\rho)\right)+1\right)}
\end{gathered}
$$

Similar to the previous scenarios, an analytical solution from (34) is not possible. However, under high SNR assumption, we have $K_{t}=K_{d}$ (see Appendix) and (34) can be simplified as

$\Phi_{T N P, I I I}=\frac{2 K_{d} \rho(1-\rho)\left(1+\left(1-K_{d}\right) G_{S R}\right)}{\left(\frac{\rho}{T_{d}}+(1-\rho)\right)}\left(\frac{P T}{N_{0} T_{d}}\right)$

For a fixed $\rho$, the optimum value of $K_{d}$ which maximizes (35) can be found by taking the derivative of (35) and solving for $K_{d}$. This yields

$$
K_{d_{-} o p t}=K_{t_{-} o p t}=\frac{1+G_{S R}}{2 G_{S R}}
$$

which takes values within the range of 0.5 and 1 . After inserting (36) into (35), we take the derivative with respect to $\rho$ and solve for $\rho_{\text {opt }}$ yielding

$$
\rho_{\text {opt }}=\left\{\begin{array}{l}
1 / 2 \quad \text { for } \quad T_{d}=1 \\
\frac{T_{d}}{T_{d}-1}\left(1-\sqrt{\frac{1}{T_{d}}}\right) \quad \text { for } \quad T_{d}>1 .
\end{array}\right.
$$

In Table II, we present optimum values of $\rho$ for $T=100$. Similar to the two previous scenarios, we have $\rho_{\text {opt }} \sim 0.9$ for $T_{t}=1$ and $\rho_{o p t}$ slightly decreases with increasing values of $T_{t}$. As for the calculation of $K_{d_{-} o p t}$ and $K_{t_{-} o p t}$ through (36), we need $G_{S R}$ which can be solved from cosine theorem for the particular relay location. Replacing it in (36), we find $K_{d_{-} \_p t}$ and $K_{t_{-} o p t}$ as 0.625 .

\section{OPTIMIZATION OF $\rho_{S}$ AND $\rho_{R}$ UNDER INP CONSTRAINT}

Under INP constraint, we have introduced two optimization parameters, namely $\rho_{S}$ and $\rho_{R}$. Here, we optimize these two parameters to maximize the average received SNR at the destination terminal. Following a similar notation in Section $\mathrm{IV}$, we formulate the optimization problem as

$$
\max _{\rho_{S}, \rho_{R}} \Phi_{I N P}\left(\rho_{S}, \rho_{R}\right) \quad \text { s.t. } \quad 0<\rho_{S}, \rho_{R}<1
$$

where the objective function $\Phi_{I N P}\left(\rho_{S}, \rho_{R}\right)$ is given by

$$
\begin{aligned}
& \Phi_{I N P}=\frac{\left(1-\rho_{S}\right) \rho_{S}\left(P_{S} T\right)^{2}}{\rho_{S} P_{S} T N_{0}+\left(1-\rho_{S}\right) P_{S} T T_{d} N_{0}+T_{d} N_{0}^{2}} \\
& +\frac{\left(1-\rho_{R}\right) \rho_{R} B_{t} B_{d}\left(P_{R} T\right)^{2}}{B_{d} \rho_{R} P_{R} T N_{0}+B_{t}\left(1-\rho_{R}\right) P_{R} T T_{d} N_{0}+T_{d} N_{0}^{2}} .
\end{aligned}
$$

A general analytic solution for (39) seems to be intractable. Therefore, in the following, we investigate two representative scenarios where the relay is either close to the source or the destination to obtain closed-form solutions. 


$$
\Phi_{T N P, I I}=\frac{\frac{8 K_{d}\left(1-K_{d}\right) \rho(1-\rho)\left(P T / N_{0}\right)^{3}}{T_{d}}\left[\frac{\rho}{T_{d}}+(1-\rho)\right]+\frac{4 \rho(1-\rho)\left(P T / N_{0}\right)^{2}}{T_{d}}-\frac{8 K_{d}\left(1-K_{d}\right) \rho(1-\rho)\left(P T / N_{0}\right)^{2}}{T_{d}}}{\left(\frac{\rho\left(P T / N_{0}\right)}{T_{d}}+(1-\rho)\left(P T / N_{0}\right)\right)\left[4 K_{d}\left(1-K_{d}\right)\left(P T / N_{0}\right)\left(\frac{\rho}{T_{d}}+(1-\rho)\right)+2\right]+1}
$$

TABLE III

Optimum $\rho_{S}, \rho_{R}$ VALUES FOR VARIOUS $T$ VALUES UNDER INP CONSTRAINT $\left(T=100, \quad \theta=\pi, \quad \alpha=2, \quad S_{S R}=S_{R D}\right)$.

\begin{tabular}{|c|c|c|c|c|c|}
\hline$P / N_{0}$ & $T_{t}=1$ & $T_{t}=5$ & $T_{t}=20$ & $T_{t}=50$ & $T_{t}=80$ \\
\hline \multicolumn{6}{|c|}{ Scenario 1: Relay is close to destination $(\beta=-30 \mathrm{~dB})$} \\
\hline $0 \mathrm{~dB}$ & $\begin{array}{c}0.8764 / 0.8764 \\
(0.8772 / 0.9086)\end{array}$ & $\begin{array}{c}0.8752 / 0.8752 \\
(0.8760 / 0.8959)\end{array}$ & $\begin{array}{c}0.8701 / 0.8701 \\
(0.8707 / 0.8553)\end{array}$ & $\begin{array}{c}0.8530 / 0.8530 \\
(0.8536 / 0.7805)\end{array}$ & $\begin{array}{c}0.8040 / 0.8040 \\
(0.8043 / 0.6754)\end{array}$ \\
\hline $5 \mathrm{~dB}$ & $\begin{array}{c}0.8969 / 0.8969 \\
(0.8972 / 0.9087)\end{array}$ & $\begin{array}{c}0.8954 / 0.8954 \\
(0.8957 / 0.9024)\end{array}$ & $\begin{array}{c}0.8889 / 0.8889 \\
(0.8893 / 0.8797)\end{array}$ & $\begin{array}{c}0.8681 / 0.8681 \\
(0.8683 / 0.8305)\end{array}$ & $\begin{array}{c}0.8129 / 0.8129 \\
(0.8130 / 0.7494)\end{array}$ \\
\hline $10 \mathrm{~dB}$ & $\begin{array}{c}0.9047 / 0.9047 \\
(0.9048 / 0.9087)\end{array}$ & $\begin{array}{c}0.9031 / 0.9031 \\
(0.9032 / 0.9053)\end{array}$ & $\begin{array}{c}0.8960 / 0.8960 \\
(0.8961 / 0.8921)\end{array}$ & $\begin{array}{c}0.8735 / 0.8735 \\
(0.8736 / 0.8584)\end{array}$ & $\begin{array}{c}0.8158 / 0.8158 \\
(0.8159 / 0.7907)\end{array}$ \\
\hline $15 \mathrm{~dB}$ & $\begin{array}{c}0.9074 / 0.9074 \\
(0.9074 / 0.9087)\end{array}$ & $\begin{array}{c}0.9057 / 0.9057 \\
(0.9057 / 0.9064)\end{array}$ & $\begin{array}{c}0.8983 / 0.8983 \\
(0.8984 / 0.8970)\end{array}$ & $\begin{array}{c}0.8753 / 0.8753 \\
(0.8753 / 0.8700)\end{array}$ & $\begin{array}{c}0.8168 / 0.8168 \\
(0.8168 / 0.8081)\end{array}$ \\
\hline $20 \mathrm{~dB}$ & $\begin{array}{c}0.9083 / 0.9083 \\
(0.9083 / 0.9087)\end{array}$ & $\begin{array}{c}0.9066 / 0.9066 \\
(0.9066 / 0.9068)\end{array}$ & $\begin{array}{c}0.8991 / 0.8991 \\
(0.8991 / 0.8986)\end{array}$ & $\begin{array}{c}0.8758 / 0.8758 \\
(0.8758 / 0.8741)\end{array}$ & $\begin{array}{c}0.8171 / 0.8171 \\
(0.8171 / 0.8143)\end{array}$ \\
\hline $25 \mathrm{~dB}$ & $\begin{array}{c}0.9085 / 0.9085 \\
(0.9086 / 0.9087)\end{array}$ & $\begin{array}{c}0.9068 / 0.9068 \\
(0.9068 / 0.9069)\end{array}$ & $\begin{array}{c}0.8993 / 0.8993 \\
(0.8993 / 0.8992)\end{array}$ & $\begin{array}{c}0.8760 / 0.8760 \\
(0.8760 / 0.8755)\end{array}$ & $\begin{array}{c}0.8172 / 0.8172 \\
(0.8172 / 0.8163)\end{array}$ \\
\hline \multicolumn{6}{|c|}{ Scenario 2: Relay is close to source $(\beta=30 \mathrm{~dB})$} \\
\hline $0 \mathrm{~dB}$ & $\begin{array}{c}0.8764 / 0.8764 \\
(0.8764 / 0.8778)\end{array}$ & $\begin{array}{c}0.8752 / 0.8752 \\
(0.8753 / 0.8768)\end{array}$ & $\begin{array}{c}0.8701 / 0.8701 \\
(0.8700 / 0.8715)\end{array}$ & $\begin{array}{c}0.8530 / 0.8530 \\
(0.8528 / 0.8540)\end{array}$ & $\begin{array}{c}0.8040 / 0.8040 \\
(0.8037 / 0.8045)\end{array}$ \\
\hline $5 \mathrm{~dB}$ & $\begin{array}{c}0.8969 / 0.8969 \\
(0.8969 / 0.8975)\end{array}$ & $\begin{array}{l}0.8954 / 0.8954 \\
(0.8954 / 0.8960)\end{array}$ & $\begin{array}{l}0.8889 / 0.8889 \\
(0.8889 / 0.8895)\end{array}$ & $\begin{array}{c}0.8681 / 0.8681 \\
(0.8680 / 0.8685)\end{array}$ & $\begin{array}{c}0.8129 / 0.8129 \\
(0.8128 / 0.8130)\end{array}$ \\
\hline $10 \mathrm{~dB}$ & $\begin{array}{c}0.9047 / 0.9047 \\
(0.9047 / 0.9050)\end{array}$ & $\begin{array}{c}0.9031 / 0.9031 \\
(0.9031 / 0.9033)\end{array}$ & $\begin{array}{c}0.8960 / 0.8960 \\
(0.8959 / 0.8961)\end{array}$ & $\begin{array}{c}0.8735 / 0.8735 \\
(0.8735 / 0.8736)\end{array}$ & $\begin{array}{c}0.8158 / 0.8158 \\
(0.8158 / 0.8159)\end{array}$ \\
\hline $15 \mathrm{~dB}$ & $\begin{array}{c}0.9074 / 0.9074 \\
(0.9074 / 0.9074)\end{array}$ & $\begin{array}{c}0.9057 / 0.9057 \\
(0.9057 / 0.9058)\end{array}$ & $\begin{array}{c}0.8983 / 0.8983 \\
(0.8983 / 0.8984)\end{array}$ & $\begin{array}{c}0.8753 / 0.8753 \\
(0.8753 / 0.8753)\end{array}$ & $\begin{array}{c}0.8168 / 0.8168 \\
(0.8168 / 0.8168)\end{array}$ \\
\hline $20 \mathrm{~dB}$ & $\begin{array}{c}0.9083 / 0.9083 \\
(0.9082 / 0.9082)\end{array}$ & $\begin{array}{c}0.9066 / 0.9066 \\
(0.9066 / 0.9066)\end{array}$ & $\begin{array}{c}0.8991 / 0.8991 \\
(0.8991 / 0.8991)\end{array}$ & $\begin{array}{c}0.8758 / 0.8758 \\
(0.8758 / 0.8758)\end{array}$ & $\begin{array}{c}0.8171 / 0.8171 \\
(0.8171 / 0.8171)\end{array}$ \\
\hline $25 \mathrm{~dB}$ & $\begin{array}{c}0.9085 / 0.9085 \\
(0.9085 / 0.9086)\end{array}$ & $\begin{array}{c}0.9068 / 0.9068 \\
(0.9068 / 0.9068)\end{array}$ & $\begin{array}{c}0.8993 / 0.8993 \\
(0.8993 / 0.8993)\end{array}$ & $\begin{array}{c}0.8760 / 0.8760 \\
(0.8760 / 0.8760)\end{array}$ & $\begin{array}{c}0.8172 / 0.8172 \\
(0.8172 / 0.8172)\end{array}$ \\
\hline
\end{tabular}

Numbers in parenthesis are obtained through numerical optimization of exact objective function given by (39).

\section{A. Scenario I: Relay is Close to Destination}

When the relay is located close to the destination node, we have $G_{S R} \approx 1$ and $G_{R D}>>1$. Therefore, we can use the approximations $B_{d} \approx P_{d, S} / P_{d, R}$ and $B_{t} \approx P_{t, S} / P_{t, R}$. Inserting these in (39), we have

$$
\Phi_{I N P, I} \approx \frac{2\left(1-\rho_{S}\right) \rho_{S}\left(P_{S} T\right)^{2}}{\rho_{S} P_{S} T N_{0}+\left(1-\rho_{S}\right) P_{S} T_{t} T_{d} N_{0}+T_{d} N_{0}^{2}} .
$$

This shows that the objective function does not depend on $\rho_{R}$ and is only a function of $\rho_{S}$. Then the optimum $\rho_{S}$ value can be found as

$$
\begin{aligned}
\rho_{S_{-} \text {opt }} & =\frac{\left(P_{S} T+N_{0}\right) T_{d}}{\left(T_{d}-1\right) P_{S} T} \\
- & \frac{\sqrt{T_{d} T^{2} P_{S}^{2}+\left(T_{d}+1\right) N_{0} T_{d} T P_{S}+N_{0}^{2} T_{d}^{2}}}{\left(T_{d}-1\right) P_{S} T} .
\end{aligned}
$$

For $\beta=-30 \mathrm{~dB}$, Table III tabulates optimum values of $\rho_{S}$ for a frame length of $T=100$ assuming various number of pilot symbols, i.e., $T_{t}=1,5,20,50,80$. We consider the case of $P_{S}=P_{R}=P$ to have a fair comparison with TNP constraint. Comparison of Table II and III shows that optimum values of $\rho$ under TNP and $\rho_{S}=\rho_{R}$ under INP constraint are quite similar.

\section{B. Scenario II: Relay is Close to Source}

When the relay is located near the source node, we have $G_{R D} \approx 1$ and $G_{S R}>>1$. Therefore, $B_{d}$ and $B_{t}$ can be simplified as $B_{d} \approx G_{R D} \approx 1$ and $B_{t} \approx G_{R D} \approx 1$. Thus the objective function takes the form of

$$
\begin{aligned}
\Phi_{I N P, I I} & \approx \frac{\left(1-\rho_{S}\right) \rho_{S}\left(P_{S} T\right)^{2}}{\rho_{S} P_{S} T N_{0}+\left(1-\rho_{S}\right) P_{S} T_{t} T_{d} N_{0}+T_{d} N_{0}^{2}} \\
& +\frac{\left(1-\rho_{R}\right) \rho_{R}\left(P_{R} T\right)^{2}}{\rho_{R} P_{R} T N_{0}+\left(1-\rho_{R}\right) P_{R} T_{t} T_{d} N_{0}+T_{d} N_{0}^{2}} .
\end{aligned}
$$

The optimum values of $\rho_{S}$ can be found from (42) and $\rho_{R}$ has a similar formula as

$$
\begin{aligned}
\rho_{R_{-} o p t} & =\frac{\left(P_{R} T+N_{0}\right) T_{d}}{\left(T_{d}-1\right) P_{R} T} \\
- & \frac{\sqrt{T_{d} T^{2} P_{R}^{2}+\left(T_{d}+1\right) N_{0} T_{d} T P_{R}+N_{0}^{2} T_{d}^{2}}}{\left(T_{d}-1\right) P_{R} T} .
\end{aligned}
$$




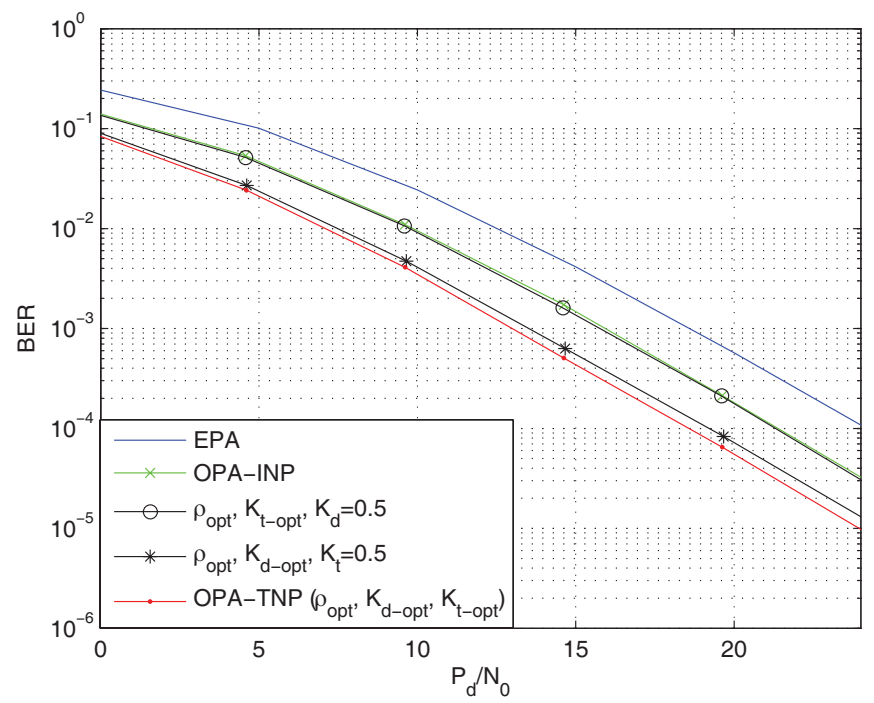

Fig. 2. BER performance of RD protocol with EPA and OPA under TNP and INP constraints for $\beta=-30 \mathrm{~dB}$.

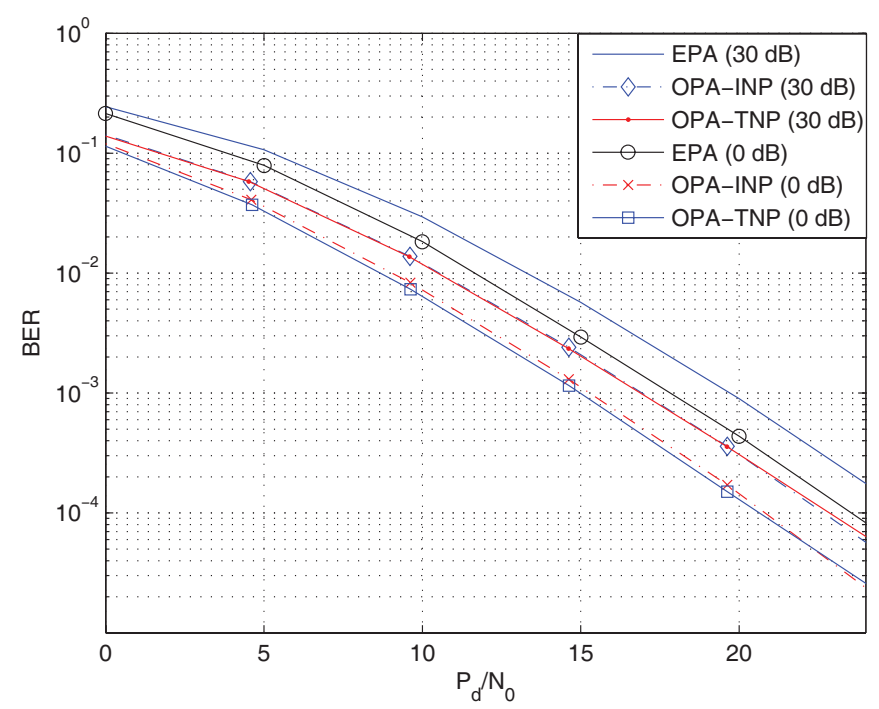

Fig. 3. BER performance of RD protocol with EPA and OPA under TNP and INP constraints for $\beta=30 \mathrm{~dB}$ and $\beta=0 \mathrm{~dB}$.

Numerical values of optimized $\rho_{S}$ and $\rho_{R}$ for $\beta=30 \mathrm{~dB}$ can be found in Table III.

\section{Simulation Results}

In this section, we present Monte Carlo simulations to demonstrate the performance of optimized scheme for different relay locations. In our simulations, we assume 4-PSK modulation, $\theta=\pi$, and $\alpha=2$. We assume $S_{R D}=S_{S R}$ unless otherwise stated. We consider a quasi-static channel with coherence time of $T_{c o h}=T$ symbols where $T$, as earlier introduced, is the frame length. For a fair comparison to TNP constraint, we set $P_{S}=P_{R}=P$ under INP constraint. The optimization parameters used in the simulation are based on the derived analytical solutions.

In Fig. 2, we present the bit error rate (BER) performance of OPA under TNP and INP constraints for $\beta=-30 \mathrm{~dB}$ along with EPA. EPA corresponds to the case when we have $\left(P_{d}=\right.$ $P_{t}=P$ and $\left.K_{d}=K_{t}=0.5\right)$ under TNP constraint and $\left(P_{S}=\right.$

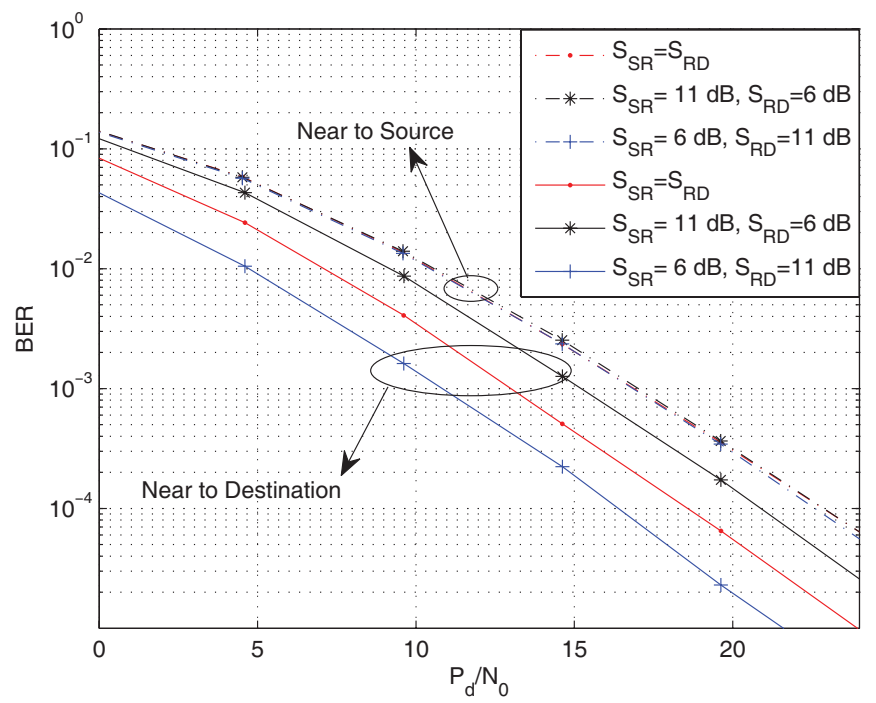

Fig. 4. BER performance of RD protocol with OPA under TNP for different shadowing effects.

$P_{R}=P$ and $\left.\rho_{S}=\rho_{R}=T_{d} / T^{3}\right)$ under INP constraint. We assume a transmission frame of $T=100$ and deployment of a single pilot symbol, i.e., $T_{t}=1$. From Fig. 2 , we observe that OPA under INP constraint brings a performance improvement of $2.8 \mathrm{~dB}$ at a target BER of $10^{-3}$ with respect to EPA ${ }^{4}$. The performance improvement climbs up to $5.5 \mathrm{~dB}$ under TNP constraint.

To further test how $K_{t}$ and $K_{d}$ values individually affect the BER performance under TNP constraint, we also investigate the following two cases where we set $\left(\rho=\rho_{\text {opt }}, K_{t}=0.5\right.$, $\left.K_{d}=K_{d, o p t}\right)$ and $\left(\rho=\rho_{\text {opt }}, K_{t}=K_{t, o p t}, K_{d}=0.5\right)$. We include the corresponding performance of these cases in Fig. 2. The results demonstrate that the first case (i.e., $K_{t}=0.5$ ) results in a minor degradation of $0.3 \mathrm{~dB}$ with respect to OPA scheme while the second case (i.e., $K_{d}=0.5$ ) results in a performance loss of $2.6 \mathrm{~dB}$. Therefore, we conclude that optimization of $K_{d}$ plays a more significant role in performance improvement.

In Fig. 3, we consider $\beta=30 \mathrm{~dB}$ (i.e., relay is located close to the source) and $\beta=0 \mathrm{~dB}$ (i.e., relay is at midway between the source and the destination) and present BER for EPA and OPA under TNP and INP constraints. At a target BER of $10^{-3}$, we observe performance improvements of 2.7 $\mathrm{dB}$ and $3.3 \mathrm{~dB}$ for $\beta=30 \mathrm{~dB}$ and $\beta=0 \mathrm{~dB}$, respectively under TNP constraint. Different from the case of $\beta=-30$ $\mathrm{dB}$ (c.f. Fig. 2) where performance improvements under two constraints significantly differ from each other, improvements are similar for these relay locations. This is rather expected for $\beta=30 \mathrm{~dB}$ where TNP and INP constraints become identical under the considered scenario.

In Fig. 4, we study the effect of shadowing in the underlying links on the system performance. We assume TNP constraint and consider two scenarios where the relay is located either close to the source or the destination. For each scenario, we consider three different shadowing values: 1) identical

\footnotetext{
${ }^{3}$ Note the definitions of $P_{d, S}=\rho_{S} P_{S} T / T_{d}$ and $P_{d, R}=\rho_{R} P_{R} T / T_{d}$.

${ }^{4}$ Since the objective function given by (40) for this particular relay location does not depend on $\rho_{R}$, we set $\rho_{R}=\rho_{S}$ in the simulations.
} 


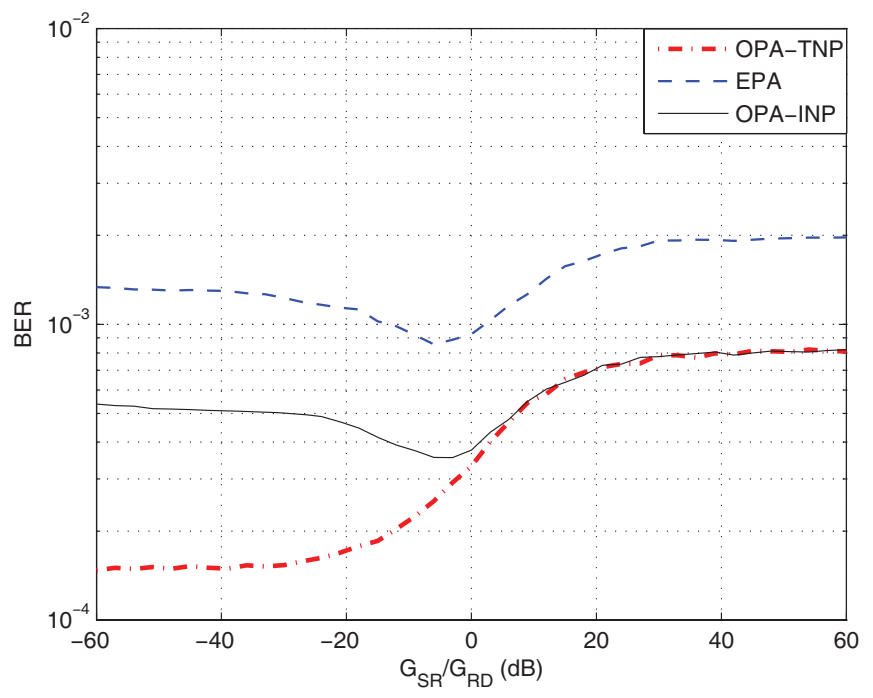

Fig. 5. BER performance of RD protocol with respect to relay location.

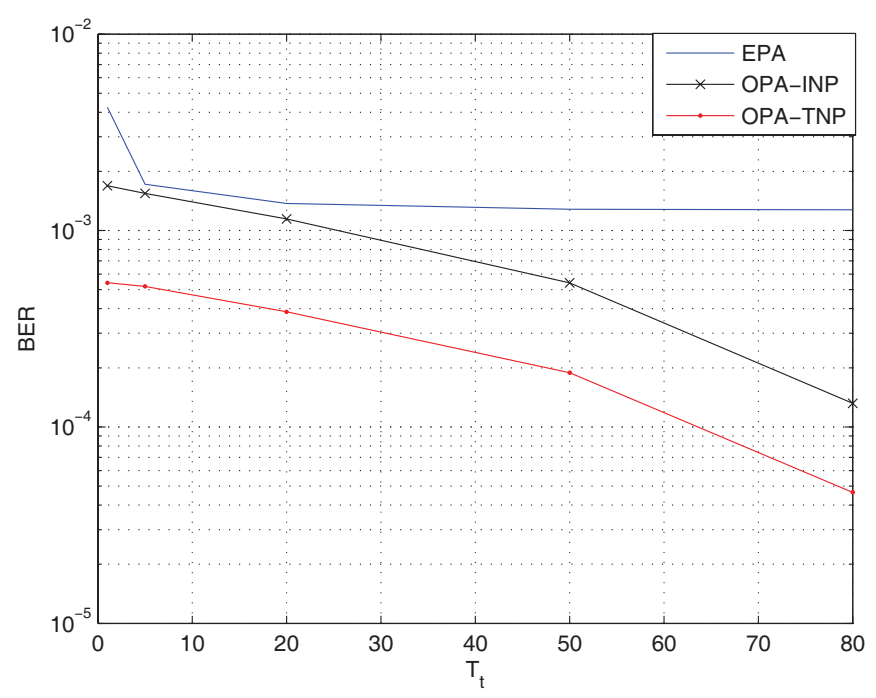

Fig. 6. Effect of training length $T_{t}$ on the performance.

power loss in $\mathrm{S} \rightarrow \mathrm{R}$ and $\mathrm{R} \rightarrow \mathrm{D}$ links due to shadowing $\left.\left(S_{R D}=S_{S R}=11 \mathrm{~dB}\right) 2\right) S_{R D}=6 \mathrm{~dB}, S_{S R}=11 \mathrm{~dB}$, 3) $S_{R D}=11 \mathrm{~dB}, S_{S R}=6 \mathrm{~dB}$ [18]. We observe that when the relay is near to the source, shadowing effect introduces negligible degradation. However, when the relay is near to the destination, performance is affected by $\pm 2 \mathrm{~dB}$ with respect to the case where identical shadowing is experienced in the underlying links.

In Fig. 5, we investigate the impact of relay location on the performance. We fix $P / N_{0}$ to $18 \mathrm{~dB}$ and present BER performance for EPA and OPA under TNP and INP constraints as a function of relay location. From Fig. 5, we observe that the error rate of OPA-INP takes its minimum value for $\beta$ around $-6 \mathrm{~dB}$. As for OPA-TNP, better performance is observed for negative values. This indicates that larger optimization gains are available as the relay node moves closer to the destination under TNP constraint confirming our earlier observations in Figs. 2 and 3.

In Fig. 6, we study the effect of training length $T_{t}$ on the BER performance. We assume $\beta=-30 \mathrm{~dB}$, frame length

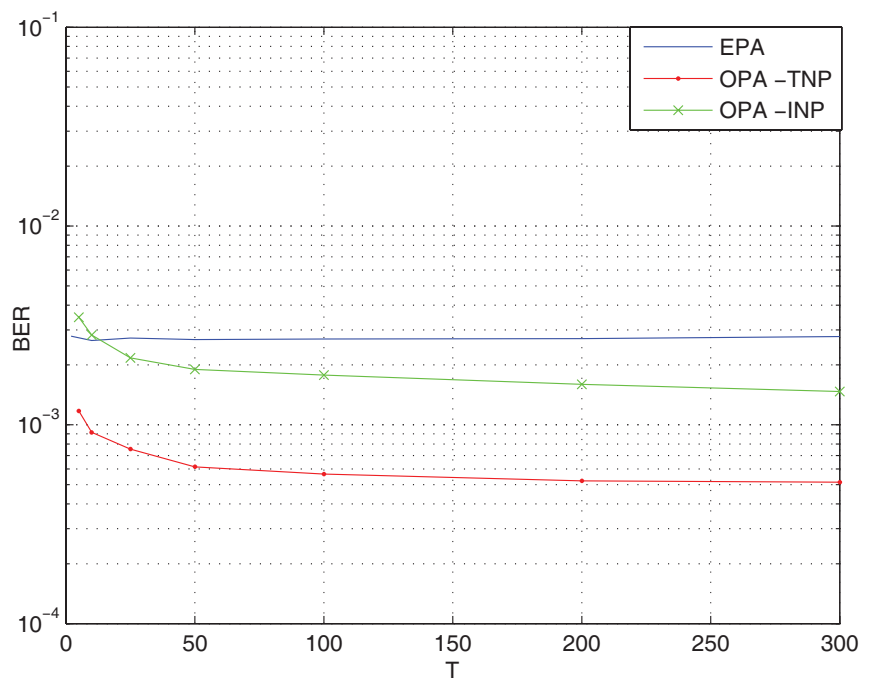

Fig. 7. Effect of frame length $T$ on the performance.

of $T=100$, and a $P / N_{0}$ value of $15 \mathrm{~dB}$. From Fig. 6, we observe that BER performance of EPA scheme first improves as $T_{t}$ increases. However, after a certain number of pilots, decoding performance at the receiver is mainly determined by data power, not by channel estimation quality. As for OPA, there is a steady improvement in BER with increasing $T_{t}$. However, it should be noted that this comes with a sacrifice of data throughput. For $\beta=30 \mathrm{~dB}$ and $\beta=0 \mathrm{~dB}$, we have made similar observations, however we have omitted those results due to space limitations.

In Fig. 7, we study the effect of frame length of $T$ on the BER performance. We assume $\beta=-30 \mathrm{~dB}$, deployment of a single pilot $\left(T_{t}=1\right)$, and $P / N_{0}=15 \mathrm{~dB}$. From Fig. 7, we observe that there is slight improvement up to $T=50$. This is as a result of the increasing training power (proportional to $T$ ) which results in smaller channel estimation errors, therefore, leading to less bit errors at the decoder. However, further increase in training power does not significantly affect error rate performance and it becomes constant regardless of frame length. Because after a certain training power is allocated, decoding performance is mainly determined by data power [24].

\section{CONCLUSION}

In this paper, we have investigated optimum power allocation for an $\mathrm{AaF}$ relaying cooperative system with channel estimation. Based on the maximization of the average received SNR at the destination node, we have formulated an optimization problem under TNP constraint to determine the allocation of total power between training and data periods as well as allocation of training/data powers between broadcasting and relaying phases. Under INP constraint, we have determined the optimum method (in the sense of SNR maximization) to allocate the transmit power between training and data transmission periods.

Under the assumption of single pilot symbol deployment and a frame length of 100 symbols, we have found out that allocating $\sim 10 \%$ of the total power to training phase would be sufficient to optimize the performance. Exact value depends 
on employed SNR and relay location. For scenarios where the relay is very close to the destination, we have found out that almost all of the available training/data power should be allocated for broadcasting phase under TNP constraint. As the relay moves away from the destination, power allocated to broadcasting phase should decrease. Eventually, when the relay is very close to the source, power allocated to broadcasting and relaying phases becomes equal to each other. We have further conducted an extensive Monte-Carlo simulation to study the effect of optimization on the error rate performance. Our simulation results demonstrate that performance gains within the range of 2.8-5.5 dB are observed for TNP constraint depending on the relay location. Most performance gains are obtained when the relay is close to the destination. The performance under INP constraint is similar to that under TNP when the relay is close to the source or at midway, however becomes inferior for scenarios when the relay comes close to the destination.

\section{APPENDIX A}

In this appendix, we demonstrate that optimization parameters $K_{d}$ and $K_{t}$ take identical values under high SNR assumption. First note that the average received SNR given by (25) can be rewritten as

$$
\begin{aligned}
\Phi_{T N P}= & \frac{\frac{4 K_{d} K_{t} \rho(1-\rho)\left(P T / N_{0}\right)}{T_{d}}}{\left(2 K_{d} \frac{\rho P T}{T_{d} N_{0}}+2 K_{t} \frac{(1-\rho) P T}{N_{0}}+1\right)}\left(P T / N_{0}\right) \\
& +\frac{\frac{B_{d} B_{t} \rho(1-\rho)\left(P T / N_{0}\right)}{T_{d}}}{\left(B_{d} \frac{\rho P T}{T_{d} N_{0}}+B_{t} \frac{(1-\rho) P T}{N_{0}}+1\right)}\left(P T / N_{0}\right)
\end{aligned}
$$

which can be approximated, under high SNR assumption, as

$$
\begin{aligned}
\Phi_{T N P} & \approx \frac{2 K_{d} K_{t} \rho(1-\rho)}{K_{d} \rho+K_{t} T_{d}(1-\rho)}\left(P T / N_{0}\right) \\
& +\frac{B_{d} B_{t} \rho(1-\rho)}{B_{d} \rho+B_{t} T_{d}(1-\rho)}\left(P T / N_{0}\right) .
\end{aligned}
$$

By taking the derivative of $\Phi_{T N P}$ with respect to $K_{t}$ and equating the resulting expression to zero yields

$$
\frac{2}{\left(K_{d} \rho+K_{t} T_{d}(1-\rho)\right)^{2}}+\frac{\left(\frac{B_{d}}{K_{d}}\right)^{2} \frac{d B_{t}}{d K_{t}}}{\left(B_{d} \rho+B_{t} T_{d}(1-\rho)\right)^{2}}=0 .
$$

Similarly, performing derivative of $\Phi_{T N P}$ with respect to $K_{d}$ and equating the resulting expression to zero, we have

$$
\frac{2}{\left(K_{d} \rho+K_{t} T_{d}(1-\rho)\right)^{2}}+\frac{\left(\frac{B_{t}}{K_{t}}\right)^{2} \frac{d B_{d}}{d K_{d}}}{\left(B_{d} \rho+B_{t} T_{d}(1-\rho)\right)^{2}}=0 .
$$

Comparison of (46) and (47) readily reveals that

$$
\left(\frac{B_{t}}{K_{t}}\right)^{2} \frac{d B_{d}}{d K_{d}}=\left(\frac{B_{d}}{K_{d}}\right)^{2} \frac{d B_{t}}{d K_{t}}
$$

Now consider scenario II where $B_{d} \approx 2-2 K_{d}$, and $B_{t} \approx$ $2-2 K_{t}$. Using these values in (48) yields

$$
\frac{1-K_{t}}{K_{t}}=\frac{1-K_{d}}{K_{d}} .
$$

Eq. (49) shows that $K_{t}=K_{d}$. For scenario III, we have $B_{d} \approx$ $2 K_{d}\left(1-K_{d}\right) G_{S R}$, and $B_{t} \approx 2 K_{t}\left(1-K_{t}\right) G_{S R}$, therefore it can be shown in a similar way that $K_{t}=K_{d}$.

\section{REFERENCES}

[1] A.Sendonaris, E. Erkip, and B. Aazhang, "User cooperation diversitypart I: system description," IEEE Trans. Commun., vol. 51, pp. 19271938, Nov. 2003.

[2] J. N. Laneman and G. W. Wornell, "Distributed space-time-coded protocols for exploiting cooperative diversity in wireless networks," IEEE Trans. Inform. Theory, vol. 49, no. 10, pp. 2451-2425, Oct. 2003.

[3] R. Nabar, H. Bolcskei, and F. Kneubuhler, "Fading relay channel: performance limits and space-time signal design," IEEE J. Select. Areas Commun., vol. 22, pp. 1099-1109, Aug. 2004.

[4] D. S. Michalopoulos, G. K. Karagiannidis, T. A. Tsiftsis, and R. K. Mallik, "Distributed transmit antenna selection (DTAS) under performance or energy consumption constraints," IEEE Trans. Wireless Commun., vol. 7, no. 4, pp. 1168-1173, Apr. 2008.

[5] D. S. Michalopoulos and G. K. Karagiannidis, "PHY-layer fairness in amplify and forward cooperative diversity systems," IEEE Trans. Wireless Commun., vol. 7, no. 3, pp. 1073-1083, Mar. 2008.

[6] M. Guizani (ed.), Wireless Communications Systems and Networks. Springer, 2004, pp. 445.

[7] H. Mheidat and M. Uysal, "Impact of receive diversity on the performance of amplify-and-forward relaying under APS and IPS power constraints," IEEE Commun. Lett., vol. 10, no. 6, pp. 468-470, June 2006.

[8] G. K. Karagiannidis, "Performance bounds of multihop wireless communications with blind relays over generalized fading channels," IEEE Trans. Wireless Commun., vol. 5, pp. 498-503, Mar. 2006.

[9] H. Mheidat and M. Uysal, "Non-coherent and mismatched-coherent receivers for distributed STBCs with amplify-and-forward relaying," IEEE Trans. Wireless Commun., vol. 6, no. 11, pp 4060-4070, Nov. 2007.

[10] C. S. Patel and G. L. Stuber, "Channel estimation for amplify and forward relay based cooperation diversity systems," IEEE Trans. Wireless Commun., vol. 6, no. 6, pp. 2348-2356, June 2007.

[11] W. Cho, R. Cao, and L. Yang, "Optimum resource allocation for amplify-and-forward relay networks with differential modulation," IEEE Trans. Signal Processing, vol. 56, no. 11, pp. 5680-5691, Nov. 2008.

[12] B. Wang, J. Zhang, and L. Zheng, "Achievable rates and scaling laws of power-constrained wireless sensory relay networks," IEEE Trans. Inform. Theory, vol. 52, no. 9, pp 4084-4104, Sept. 2006.

[13] G. K. Karagiannidis, T. A. Tsiftsis, and R. K. Mallik, "Bounds of multihop relayed communications in Nakagami-m fading," IEEE Trans. Commun., vol. 54, pp. 18-22, Jan. 2006.

[14] T. Cui, F. Gao, and A. Nallanathan, "Optimal training design for channel estimation in amplify and forward relay networks," in Proc. IEEE Globecom, Nov. 2007.

[15] J. N. Laneman, D. N. C. Tse, and G. W. Wornell, "Cooperative diversity in wireless networks: efficient protocols and outage behavior," IEEE Trans. Inform. Theory, vol. 50, no. 12, pp. 3062-3080, Dec. 2004.

[16] J. Zhang and M. C. Gursoy, "Achievable rates and optimal resource allocation for imperfectly known relay channels," in Proc. 45th Annual Allerton Conf. on Commun., Sept. 2007.

[17] H. Ochiai, P. Mitran, and V. Tarokh, "Design and analysis of collaborative diversity protocols for wireless sensor networks," in Proc. IEEE Vehicular Technology Conf., vol. 7, pp. 4645-4649, Fall 2004.

[18] K. Konstantinou, S. Kang, and C. Tzaras, "A measurement-based model for mobile-to-mobile UMTS links," in Proc. VTC Spring, Apr. 2007.

[19] J. K. Cavers, "An analysis of pilot symbol assisted modulation for Rayleigh fading channels," IEEE Trans. Veh. Technol., vol. 40, no. 4, pp. 686-693, Nov. 1991

[20] L. Tong, B. M. Sadler, and M. Dong, "Pilot-assisted wireless transmissions," IEEE Signal Processing Mag., vol. 21, no. 6, pp. 12-25, Nov. 2004.

[21] D. Chen and J. N. Laneman, "Cooperative diversity for wireless fading channels without channel state information," in Proc. Asilomar Conf. Signals, Systems and Computers, Monterey, Nov. 2004.

[22] S. M. Kay, Fundamentals of Statistical Signal Processing: Estimation Theory. Prentice-Hall, 1993.

[23] G. Taricco and E. Biglieri, "Space-time decoding with imperfect channel estimation," IEEE Trans. Wireless Commun., vol. 4, no. 4, pp. 18741888, July 2005.

[24] B. Hassibi and B. M. Hochwald, "How much training is needed in multiple antenna wireless links," IEEE Trans. Inform. Theory, vol. 49, no. 4, pp. 951-963, Apr. 2003. 


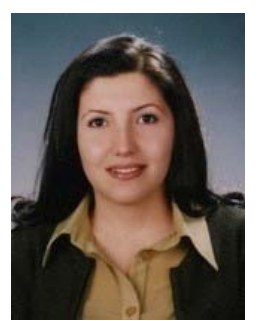

Berna Gedik was born in Ankara, Turkey in 1982. She received B.Sc. in Electrical and Electronics Engineering from Middle East Technical University (METU), Ankara, Turkey, in 2004 and M.S. in Electrical Engineering from University of Waterloo, Waterloo, Ontario, Canada in 2008. She is currently affiliated with the General Electric Company. Her research interests include cooperative communications and wireless channel estimation.

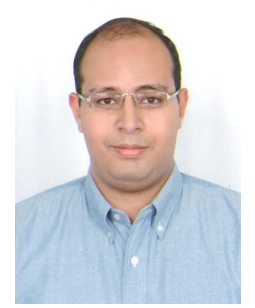

Osama Amin was born in Nancy, France in 1978. He received B.Sc. degree in Electrical and Electronics Engineering from South Valley University, Aswan, Egypt, in 2000 and M.Sc. degree in Electrical Engineering from Assiut University, Assiut, Egypt in 2004. He is currently a Ph.D. student in Electrical and Computer Engineering, University of Waterloo, Canada. His research interests include cooperative communications, adaptive systems, OFDM, and channel estimation.

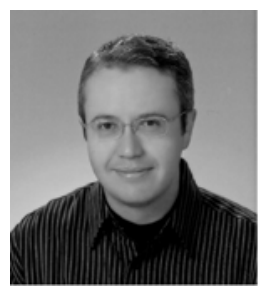

Murat Uysal was born in Istanbul, Turkey in 1973. He received the B.Sc. and the M.Sc. degree in electronics and communication engineering from Istanbul Technical University, Istanbul, Turkey, in 1995 and 1998, respectively, and the Ph.D. degree in electrical engineering from Texas A\&M University, College Station, Texas, in 2001. Since 2002, he has been with the Department of Electrical and Computer Engineering, University of Waterloo, Canada, where he is now an Associate Professor. His general research interests lie in communications theory and signal processing for communications with special emphasis on wireless applications. Specific research areas include MIMO techniques, space-time coding, diversity techniques and coding for fading channels, cooperative communications, and free-space optical communications.

Dr. Uysal is an Associate Editor for IEEE TRANSACTIONS ON WIRELESS Communications and IEEE Communications LetTers. He was a Guest Co-Editor for WILEY JOURNAL ON WIRELESS COMMUNICATIONS AND Mobile Computing's Special Issue on "MIMO Communications" published in 2004. He is currently serving as a Guest Co-Editor for IEEE JOURNAL ON SELECTED AREAS IN COMMUNICATIONS Special Issue on "Optical Wireless Communications" to be published in 2010. Over the years, he has served on the technical program committee of more than 50 international conferences in the communications area. He recently cochaired IEEE ICC'07 Communication Theory Symposium and CCECE'08 Communications and Networking Symposium. Dr. Uysal is a Senior IEEE member. 